\title{
Adult onset Still's disease accompanied by acute respiratory distress syndrome: A case report
}

\author{
XIAO-TU XI ${ }^{1}$, MAO-JIE WANG ${ }^{2}$, RUN-YUE HUANG ${ }^{2}$ and BANG-HAN DING ${ }^{1}$ \\ ${ }^{1}$ Emergency Department; ${ }^{2}$ Rheumatology Department, The Second Affiliated Hospital of Guangzhou University of \\ Chinese Medicine (Guangdong Provincial Hospital of Chinese Medicine), Guangzhou, Guangdong 510006, P.R. China
}

Received July 17, 2015; Accepted May 20, 2016

DOI: $10.3892 /$ etm.2016.3512

\begin{abstract}
Adult onset Still's disease (AOSD) is a systemic inflammatory disorder characterized by rash, leukocytosis, fever and arthralgia/arthritis. The most common pulmonary manifestations associated with AOSD are pulmonary infiltrates and pleural effusion. The present study describes a 40-year-old male with AOSD who developed fever, sore throat and shortness of breath. Difficulty breathing promptly developed, and the patient was diagnosed with acute respiratory distress syndrome (ARDS). The patient did not respond to antibiotics, including imipenem, vancomycin, fluconazole, moxifloxacin, penicillin, doxycycline and meropenem, but was sensitive to glucocorticoid treatment, including methylprednisolone sodium succinate. ARDS accompanied by AOSD has been rarely reported in the literature. In conclusion, in a patient with ARDS who does not respond to antibiotic treatment, the involvement of AOSD should be considered.
\end{abstract}

\section{Introduction}

Adult-onset Still's disease (AOSD) is a multisystemic inflammatory disorder characterized by a high spiking fever, a transient rash and arthritis/arthralgia (1). Other clinical symptoms include sore throat, myalgia, lymphadenopathies, splenomegaly and neutrophilic leukocytosis (2). The etiology of AOSD remains unclear, but an autoimmune pathogenesis has been suggested (3). The most common pulmonary manifestations associated with AOSD are pulmonary infiltrates and pleural effusion (4). However, acute respiratory distress syndrome (ARDS) accompanied by AOSD has rarely been reported (5-7). ARDS is a form of acute diffuse lung injury characterized by severe inflammation, increased pulmonary

Correspondence to: Dr Bang-Han Ding, Emergency Department, The Second Affiliated Hospital of Guangzhou University of Chinese Medicine (Guangdong Provincial Hospital of Chinese Medicine), 111 Dade Road, Guangzhou, Guangdong 510006, P.R. China E-mail: banghanding@139.com

Key words: adult onset Still's disease, acute respiratory distress syndrome, spiking fever vascular permeability and a loss of aerated lung tissue (8). ARDS occurs in 7.2-38.9/100,000 individuals $(9,10)$, and has a mortality rate of $\sim 40 \%$ (11). Patient mortality varies based on numerous factors, including age, etiology of the lung injury and non-pulmonary organ dysfunction. The most common risk factors for ARDS are pneumonia, sepsis, aspiration and trauma (11). Multiple predisposing risk factors, in addition to some secondary factors such as alcohol abuse and obesity, also increase the risk (4). In addition to patient support provided by improving oxygen delivery to the tissues and preventing multiorgan system failure, early recognition and correction of the underlying cause is important in ARDS treatment. A thorough patient assessment searching for the underlying cause should be undertaken; once identified, it should be managed appropriately. Potential sources of infection should also be sought to initiate timely and appropriate antibiotic therapy to improve sepsis-associatted outcomes $(4,11)$. ARDS accompanied by AOSD is sensitive to high dose corticosteroid therapy. The present study reports the case of a 40-year old male diagnosed with AOSD accompanied by ARDS.

\section{Case report}

A 40-year-old Chinese male was admitted to The Second Affiliated Hospital of Guangzhou University of Chinese Medicine (Guangzhou, China) with a four-day history of intermittent fever accompanied by with shortness of breath for one day. The patient was a driver who did not smoke or drink, and had no recent travel or contact history of infectious diseases. On June 3, 2014, the patient had a temperature of $39^{\circ} \mathrm{C}$ and developed fever, cough, a stuffy, runny nose and sore throat. On June 5, 2014, the patient was admitted to the Emergency Department at The Second Affiliated Hospital of Guangzhou University Chinese Hospital where laboratory analyses was performed. The patient had an initial white blood cell (WBC) count of $33.25 \times 10^{9}$ cells $/ 1$ (normal range, $4-10 \times 10^{9}$ cells $/ 1$ ) with $92.1 \%$ neutrophils (normal range, 50-70\%), and a routine urine test revealed positive urine protein (1-2 g, 2+) and leukocyte esterase (+). From these results, the patient was diagnosed with a urinary tract infection, and was treated with levofloxacin $[100 \mathrm{ml}$ (0.5 g), q.d.; Daiichi Sankyo, Co., Ltd., Shanghai, China] and antipyretic therapy (ibuprofen suspension; $20 \mathrm{ml}$, p.o., t.i.d.; Jiangsu Hengrui Medicine Co., Ltd., Jiangsu, China). 
However, the body temperature of the patient continued to fluctuate between 38.9 and $39.2^{\circ} \mathrm{C}$, accompanied by mild shortness of breath and headache.

On June 6, 2014, further examination was performed and results indicated that the respiratory status of the patient had worsened (Table I). Computerized axial tomography (CT) scanning of the abdomen showed splenomegaly and bilateral kidney inflammatory effusions (Fig. 1). As a result, the patient was diagnosed with sepsis, acute respiratory distress syndrome (ARDS), multiple organ dysfunction syndrome (including the heart, lungs and liver), infective fever (perirenal infection), fatty liver and splenomegaly. The patient was subsequently treated with tienam (1,000 mg, b.i.d.; EMD Millipore, Billerica, MA, USA), polyene phosphatidylcholine (456 mg, t.i.d.; Sanofi S.A., Paris, France), plavix (75 mg, q.d.; Sanofi S.A.), aspirin (100 mg, q.d.; Bayer AG, Leverkusen, Germany) and lipitor. However, the results obtained on the following day suggested that the patient's condition was deteriorating; therefore, the patient was immediately admitted to the Intensive Care Unit (ICU).

Following admission to ICU (June 7, 2014), the vital signs of the patient were as follows: Body temperature, $38.6^{\circ} \mathrm{C}$ (normal range, $36.0-37.0^{\circ} \mathrm{C}$ ); pulse rate, 121 beats $/ \mathrm{min}$ (normal range, $60-100$ beats/min); respiratory rate, 32 breaths/min (normal range, $70-75$ breaths/min); and blood pressure, $131 / 72 \mathrm{mmHg}$ (normal range, 90-139/60-89 mmHg). Physical examination revealed bilateral coarse breath sounds over the lung fields without dry or wet rale, mild yellow dye of the systemic skin mucous membrane and sclera, pharyngeal hyperemia, and 121 beats/min heart rate with the cardiac dull field expanded, a sign of pericardial effusion. The abdomen was soft, while the spleen could be touched below the left rib with $\sim 2$ fingers in width. In addition, the spleen felt tough with smooth edges with no haphalgesia. Laboratory test results are presented in Table II. The results of chest radiography were as follows: Pulmonary congestion, heart enlargement and a small quantity of pleural effusion on both sides. This led to a diagnosis of cardiac insufficiency and infection in the lower right lung. The therapeutic strategy comprised of ventilation using a non-invasive ventilator, anti-infection therapy using imipenem (1,000 mg, b.i.d.; EMD Millipore), stomach protection using pantoprazole, phlegm control using ambroxol, myocardial nutrition with creatine phosphate sodium, liver protection using a combination of transmetil and glycyrrhizin, and a number of other add-on treatments, such as traditional Chinese Medicine and nutritional treatments.

On the night of June 8th, 2014, the patient still felt short of breath, despite the ventilation using a non-invasive ventilator. Physical examination revealed the following: Body temperature, $39.5^{\circ} \mathrm{C}$; pulse rate, 135 beats $/ \mathrm{min}$; respiratory rate, 40 breaths/min; blood pressure, $122 / 73 \mathrm{mmHg}$; and peripheral blood oxygen, $93 \%$. The breathing of the patient was weak and scattered moist rales were observed over lung fields. A chest and abdomen $\mathrm{CT}$ was performed and the following results (Fig. 2) were obtained: i) Inflammation was present in the inferior lingular segment of the upper left lung and the dorsal and basal segments of both lungs; and ii) bilateral pleural effusion was observed. These results were severe compared with the results obtained on June 6th, 2014. In addition, segmental compression and hypoventilation were observed in the lower lungs. iii) Heart shadow was increased, primarily due to left ventricular enlargement. Furthermore, a small quantity of pericardial effusion was demonstrated; iv) splenauxe; v) increased exudation around both kidneys compared with the results of the CT scan on June 6, 2014, and a small quantity of effusion was observed in the bilateral paracolic sulci the abdomen; and vi) pelvic effusion. Furthermore, blood gas analyses $\left(\mathrm{PaO}_{2}\right.$, $66.5 \mathrm{mmHg} ; \mathrm{PaCO}_{2}, 32.1 \mathrm{mmHg}$ ) indicated that the breathing problem was severe. The patient was diagnosed with ARDS, and symptomatic treatments, including morphine and propofol treatment, were immediately performed.

Over a number of days, laboratory test results were obtained (Table II) and the therapeutic strategy was subsequently modified. Propofol and morphine were administered for sedation and analgesia, and esmolol was used to reduce the heart rate. In addition, anti-infection therapy was expanded and a number of antibiotics were administered, including imipenem, vancomycin (500 mg, q. 8 h; Eli Lilly Japan K.K., Kobe, Japan), fluconazole (400 mg, q.d.; Pfizer, Inc., New York, NY, USA ), moxifloxacin (400 mg, q.d.; Bayer AG), penicillin, doxycycline (200 mg, q.d.; Guangdong Weilun Biological Co., Ltd., Guangdong, China) and meropenem (1,000 mg, q. 8 h; Sumitomo Dainippon Pharma Co., Ltd., Osaka, Japan). Other symptomatic treatments, such as protecting the liver and stomach, nourishing the myocardium, correcting anemia and improving the immune system were administered. On June 14th, 2014, the oxygenation index was improved (>200), therefore, the trachea intubation was removed and a non-invasive ventilator for assisted ventilation was adopted. Hydrocortisone (100 mg i.v.d. b.i.d.; Tianjin Biochemical Pharmaceutical Co., Ltd., Tianjin, China) was administered for two days as the result of recurrent fever on June 20, 2014. As a result of the fever, all antibiotic treatment was discontinued on June 24, 2014. The breathing and liver function of the patient were improved, although pyrexia remained. The patient was, therefore, moved to the general inpatient area for further treatment.

Following transfer to the general inpatient area, a number of examinations were performed, and the results were as follows: Epstein-Barr virus (EBV)-DNA, 7.50×10³ IU/ml; EBV-DNA, $<13 \mathrm{IU} / \mathrm{ml}$ (re-checked at Sun Yat-sen Memorial Hospital, Sun Yat-sen University, Guangdong, China); tuberculosis T-spot check was negative; antibodies for auto-immune diseases were re-tested and were positive (1:100) for antinuclear antibodies, whereas the remaining items were negative; ferritin, $2,823 \mathrm{mg} / \mathrm{l}$. The state of illness was improved following the administration of various treatments (morphine and propofol); however, the recurrent fever did not improve. The patient's condition was confusing, as he did not respond to anti-infection treatment, and no signs of tumors or rheumatoid-associated features were observed during the disease process. Furthermore, the high fever could not be explained.

Notably, it was determined that the patient experienced a fever 10 years previously (July 19th, 1997) and had been diagnosed with allergic subsepticemia syndrome at The Second Affiliated Hospital of Guangzhou University of Chinese Medicine; the previous medical records of the patient was obtained from the medical record library. Following a careful review of the medical records, it was identified that the patient had a high fever with no response to antibiotic therapy 10 years previously; however, the fever was relieved following treatment 
Table I. Laboratory analysis results prior to admission to patient admission to intensive care.

\begin{tabular}{lccc}
\hline & \multicolumn{3}{c}{ Date } \\
\cline { 2 - 4 } Parameter & June 5 th & June 6 th & June 7 th \\
\hline White blood cell & $33.25 \times 10^{9} / 1$ & - & $25.88 \times 10^{9} / 1$ \\
Neutrophil & $92.10 \%$ & - & $94 \%$ \\
Urine protein & $2+$ (moderate $)$ & - & - \\
Urine leukocyte esterase & + (positive $)$ & - & - \\
Procalcitonin & - & $3.49 \mathrm{ng} / \mathrm{ml}$ & $3.07 \mathrm{ng} / \mathrm{ml}$ \\
Total bilirubin & - & $53.8 \mu \mathrm{mol} / \mathrm{l}$ & $0.431 \mathrm{~g} / \mathrm{l}$ \\
Bilirubin conjugated & - & $7.2 \mu \mathrm{mol} / \mathrm{l}$ & - \\
Blood urea nitrogen & - & $21.5 \mu \mathrm{mol} / \mathrm{l}$ & - \\
Arterial blood gas, $\mathrm{pO}_{2}$ & - & $72 \mathrm{mmHg}$ & $70 \mathrm{mmHg}$ \\
Arterial blood gas, $\mathrm{pCO}_{2}$ & - & $29.9 \mathrm{mmHg}$ & $30.6 \mathrm{mmHg}$ \\
Cardiac troponin I & - & $1.98 \mathrm{~g} / \mathrm{l}$ & - \\
N-terminal of the pro-hormone brain natriuretic peptide & - & $1763 \mathrm{pg} / \mathrm{l}$ & - \\
B2-microglobulin & - & - & $2.35 \mathrm{mg} / \mathrm{l}$ \\
C reactive protein & - & - & $200 \mathrm{mg} / \mathrm{l}$ \\
Erythrocyte sedimentation rate & - & - & $100 \mathrm{~mm} / \mathrm{h}$ \\
\hline
\end{tabular}

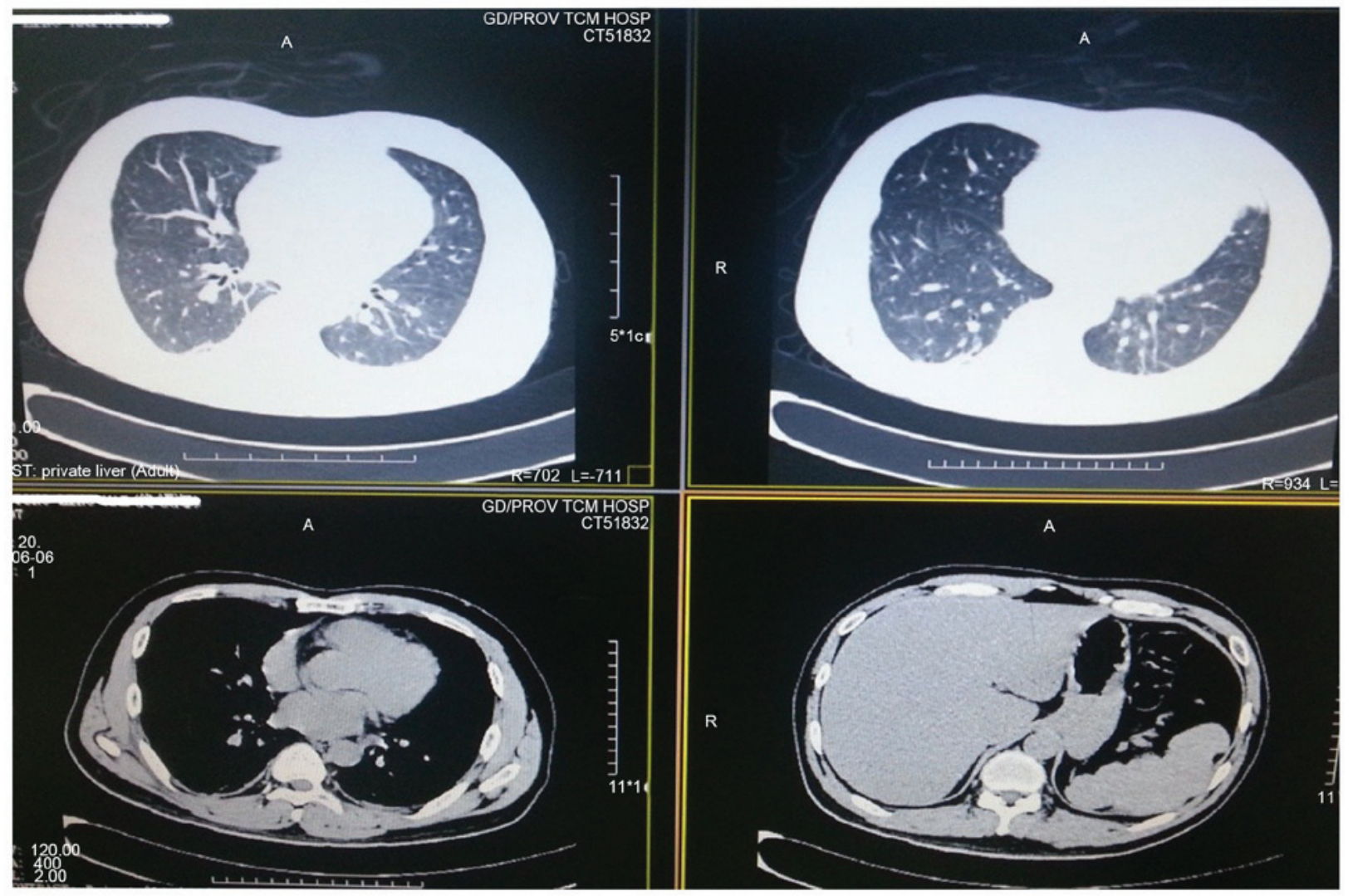

Figure 1. Upper abdominal computed tomography on June 6th 2014. Fatty liver (moderate), splenomegaly, bilateral kidney inflammatory effusions and pleural thickening of the bilateral bases of the lung can be observed.

with dexamethasone. The patient was diagnosed with AOSD according to the Yamaguchi criteria (11) in 2014. The patient in the current study met the following criteria: Fevers lasting $>1$ week; leukocytosis with neutrophilia; sore throat; temporal rash; transient arthralgia; abnormal liver enzymes; polyserositis; liver functional abnormality; splenomegaly; and negative rheumatoid factor (12). A hormone therapy program (prednisone acetate tablets administered at 40,35,30, 25, 20, 15 and $10 \mathrm{mg}$ p.o.q.d. on weeks $1-8,9,10,11,12-13,14-15$ and 16 , respectively) was therefore applied for further treatment. To date, the general 
Table II. Laboratory analysis results during patient hospitalization in the Intensive Care Unit.

\begin{tabular}{|c|c|c|c|}
\hline \multirow[b]{2}{*}{ Parameter } & \multicolumn{3}{|c|}{ Date } \\
\hline & June 7 th & June 14th & June 23rd \\
\hline White blood cell & $27.33 \times 10^{9} / 1$ & - & - \\
\hline Neutrophil & $93 \%$ & - & - \\
\hline Prothrombin time & $15.3 \mathrm{sec}$ & - & - \\
\hline International Normalised Ratio & 1.21 & - & - \\
\hline Fibrinogen & $9.23 \mathrm{~g} / 1$ & - & - \\
\hline D-dimer & $>8,000 \mu \mathrm{g} / \mathrm{l}$ & - & - \\
\hline Ferritin & $3,364 \mathrm{mg} / \mathrm{l}$ & - & - \\
\hline Arterial blood gas, $\mathrm{pO}_{2}$ & $98.6 \mathrm{mmHg}$ & - & - \\
\hline Arterial blood gas, $\mathrm{pCO}_{2}$ & $27 \mathrm{mmHg}$ & - & - \\
\hline Cardiad troponin I & $0.342 \mu \mathrm{g} / \mathrm{l}$ & - & - \\
\hline Vasculitis related antibodies & Negative & - & - \\
\hline Rheumatoid factor & Negative & - & - \\
\hline Urinary leukocyte esterase & Negative & - & - \\
\hline Urobilinogen & $4+($ severe $)$ & - & - \\
\hline Urine bilirubin & $2+($ moderate $)$ & - & - \\
\hline Leptospira antibody & - & $\begin{array}{l}\text { Positive }(+, 1: 100) \text {, indicating } \\
\text { Jaundice hemorrhagic fever }\end{array}$ & - \\
\hline Epstein-Barr viral nucleic acid & - & $+($ positive $)$ & - \\
\hline Liver fluke antibody & - & - & + \\
\hline Paragonimiasis antibody & - & - & + \\
\hline
\end{tabular}

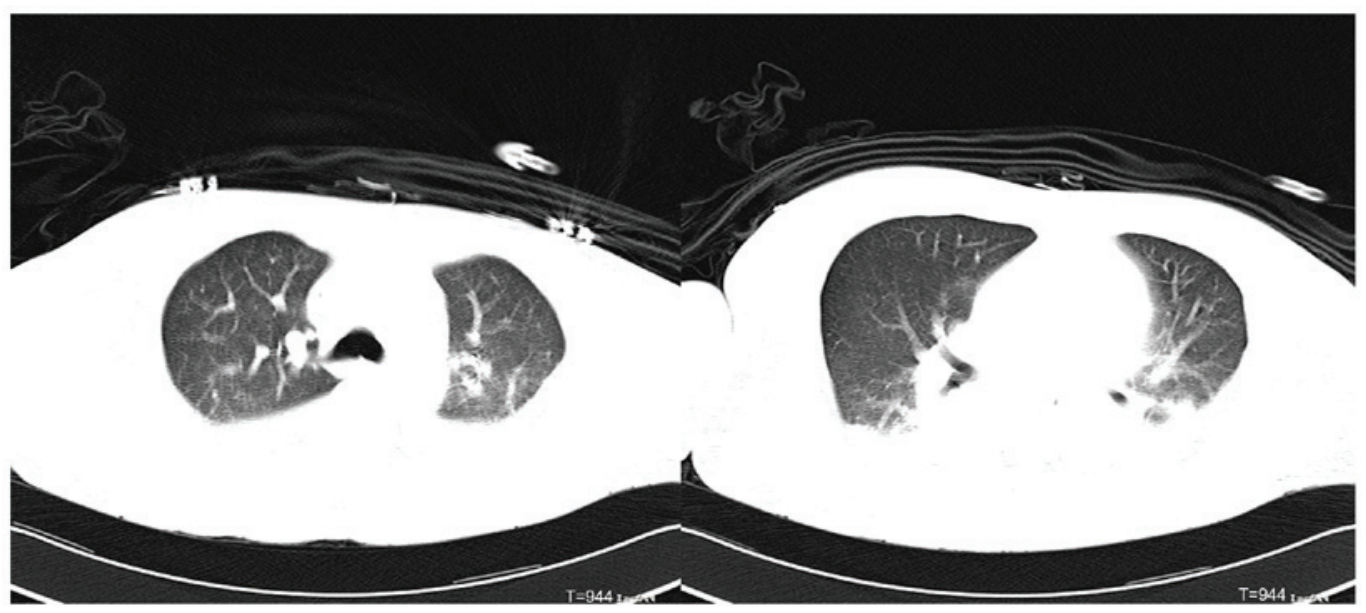

Figure 2. Upper abdominal computed tomography on June 8th 2014. The inferior lingular segment of the upper left lung, and the dorsal and basal segments of both lungs exhibited mild inflammation. Bilateral pleural effusion along with areas of atelectasis occurring in association with pressure, the effusion are more than before. The pericardium showed a small amount of effusion. Increased exudation was observed around the double kidneys, bilateral paracolic sulci in abdominal are found a little effusion either; A little effusion in Pelvic effusion in pelvic cavity.

condition of the patient is healthy and no further episodes of fever have been experienced.

\section{Discussion}

AOSD is difficult to diagnose, particularly when it presents with a number of severe systemic manifestations. In the present study, when the patient was admitted, it was difficult to obtain an accurate diagnosis; this raises two issues. First is the acute onset of dyspnea. ARDS is a severe complication associated with high morbidity and mortality (9). Saving lives is the priority in patients with acute severe ARDS. In the current study, various treatment strategies were performed to maintain the vital signs of the patient. As the clinicians focused on improving the dyspnea and preventing mortality, the diagnosis of the primary disease was initially overlooked. Secondly, the patient experienced atypical symptoms. To date, there are no specific clinical, serological and/or instrumental diagnostic 
markers for AOSD diagnosis (13). AOSD is an inflammatory disorder with unknown etiology, and is characterized by typical but non-pathognomonic clinical and laboratory features, which are commonly used to classify the disease after the exclusion of infectious, neoplastic or autoimmune disorders (14). In the current case, the clinical manifestations of the patient were not typical, and the sore throat, rash and joint pains did not persist for long periods of time. The disease developed rapidly and ARDS was induced immediately; therefore, it was difficult to obtain a diagnosis of AOSD.

To the best of our knowledge, the association between lung diseases and AOSD has been reported numerous times $(5,15,16)$, and pleuritis associated with AOSD has been widely reported, ranging between 12 and $53 \%$ in various studies $(17,18)$. Pleuritis is usually followed by pleural effusion (12). ARDS is a type of acute diffuse lung injury characterized by severe inflammation, increased pulmonary vascular permeability and a loss of aerated lung tissue (10). There is currently no effective treatment for ARDS, and management of the disease is focused on supportive therapy, avoiding complications and identifying/treating the underlying cause (19). A previous study reviewed numerous cases of ARDS in AOSD, and identified that the majority of cases studied have similar clinical and serologic symptoms (20). Similar to the case presented in the current study, those cases were initially misdiagnosed as infectious pneumonia, and broad-spectrum antibiotics were initially used to treat the patients (20); all cases showed a good response to high dose methylprednisolone therapy (20).

In conclusion, the case in the present study is similar to that experienced by other clinicians. It is important to recognize that reviewing the past medical history of a patient is required, as it can provide important information for diagnosis. In addition, although there are a number of laboratory methods available for providing clinical diagnoses, it is important to differentiate false positive data and to combine laboratory data and symptoms in order to analyze the condition of a patient carefully. Finally, to diagnose ASOD, the possibility of tumors and infectious diseases must be excluded. When a patient is not responding to anti-infectious therapy, a diagnosis of AOSD can be considered, accompanied by ARDS.

\section{Acknowledgements}

The authors are grateful to staff at the Radiology Department at The Second Affiliated Hospital of Guangzhou University of Chinese Medicine for their review of the imaging diagnosis. The authors also thank Dr Yong-Liang Chu for his assistance in the decision of the diagnosis.

\section{References}

1. Gerfaud-Valentin M, Jamilloux Y, Iwaz J and Sève P: Adult-onset Still's disease. Autoimmun Rev 13: 708-722, 2014.

2. Kim YJ, Koo BS, Kim YG, Lee CK and Yoo B. Clinical features and prognosis in 82 patients with adult-onset Still's disease. Clin Exp Rheumatol 32: 28-33, 2014.

3. Jamilloux Y, Gerfaud-Valentin M, Martinon F, Belot A, Henry T and Sève P: Pathogenesis of adult-onset Still's disease: New insights from the juvenile counterpart. Immunol Res 61: 53-62, 2015.

4. Cheema GS and Quismorio FP Jr: Pulmonary involvement in adult-onset Still's disease. Curr Opin Pulm Med 5: 305-309, 1999.

5. Dua AB, Manadan AM and Case JP. Adult onset Still's disease presenting with acute respiratory distress syndrome: Case report and review of the literature. Open Rheumatol J 7: 125-128, 2013.

6. Hagiyama H, Koike R, Nagasaka K, Nonomura Y, Nishio J, Nanki T, Kohsaka H, Kubota T and Miyasaka N: Two cases of acute respiratory distress syndrome resulting from adult-onset Still's disease. Mod Rheumatol 13: 76-80, 2003.

7. Suleiman M, Wolfovitz E, Boulman N and Levy Y: Adult onset Still's disease as a cause of ARDS and acute respiratory failure. Scand J Rheumatol 31: 181-183, 2002.

8. ARDS Definition Task Force, Ranieri VM, Rubenfeld GD, Thompson BT, Ferguson ND, Caldwell E, Fan E, Camporota L and Slutsky AS: Acute respiratory distress syndrome: The berlin definition. JAMA 307: 2526-2533, 2012.

9. Carlucci M, Graf N, Simmons JQ and Corbridge SJ: Effective management of ARDS. Nurse Pract 39: 35-40, 2014.

10. Ware LB: Pathophysiology of acute lung injury and the acute respiratory distress syndrome. Semin Respir Crit Care Med 27: 337-349, 2006

11. Yamaguchi M, Ohta A, Tsunematsu T, Kasukawa R, Mizushima Y, Kashiwagi H, Kashiwazaki S, Tanimoto K, Matsumoto Y, Ota T, et al: Preliminary criteria for classification of adult Still's disease. J Rheumatol 19: 424-430, 1992.

12. Yamaguchi M, Ohta A, Tsunematsu T, Kasukawa R, Mizushima Y, Kashiwagi H, Kashiwazaki S, Tanimoto K, Matsumoto Y, Ota T, et al: Preliminary criteria for classification of adult Still's disease. J Rheumatol 19: 424-430, 1992.

13. Manzini CU, Brugioni L, Colaci M, Tognetti M, Spinella A, Sebastiani M, Giuggioli D and Ferri C: Elevated troponin serum levels in adult onset Still's disease. Case Rep Rheumatol 2015: 732095, 2015.

14. Hu Q, Yan Z and Zhong J: Adult-onset Still's disease: How to make a diagnosis in an atypical case. Rheumatol Int 32: 3299-3302, 2012.

15. Qi H, Yin C, Xiao H and Duan T: A rare case of diffuse pulmonary nodules in a patient with adult-onset Still's disease. Intern Med 53: 1869-1872, 2014.

16. Sato H, Yokoe I, Nishio S, Onishi T, Takao T, Kobayashi Y and Haraoka H: A case of adult onset Still's disease complicated with cryptogenic organizing pneumonia. Intern Med 50: 247-251, 2011.

17. Zeng T, Zou YQ, Wu MF and Yang CD: Clinical features and prognosis of adult-onset still's disease: 61 cases from China. J Rheumatol 36: 1026-1031, 2009.

18. Ikeue T, Fukuhara A, Watanabe S, Sugita T, Horikawa S, Suzuki Y, Nishiyama $\mathrm{H}$ and Maekawa N: A case of severe adultonset Still's disease presenting with pleuropericarditis. Nihon Kokyuki Gakkai Zasshi 44: 389-393, 2006 (In Japanese).

19. Erickson SE, Martin GS, Davis JL, Matthay MA and Eisner MD; NIH NHLBI ARDS Network: Recent trends in acute lung injury mortality: 1996-2005. Crit Care Med 37: 1574-1579, 2009.

20. Hu Y, Wang H and Deng J: Adult-Onset Still's disease associated with thyroid dysfunction: Case report and review of the literature. Open Rheumatol J 8: 9-12, 2014. 